\title{
Prostatectomía radical extraperitoneal Da Vinci
}

\author{
John $\mathrm{H}^{*, * *}$, Schmid DM**, Fehr JL*. \\ *Centro de Urología, Klinik Hirslanden $y{ }^{* * C l i n i c a ~ U r o l o ́ g i c a, ~ U n i v e r s i t a ̈ t s s p i t a l ~ Z u ̈ r i c h ~}$
}

Actas Urol Esp. 2007;31(6):580-586

\section{RESUMEN}

PROSTATECTOMÍA RADICAL EXTRAPERITONEAL DA VINCI

Objetivo. Informar de la evolución de 100 prostatectomías radicales laparoscópicas asistidas por robot (RALPs), una alternativa minimamente invasiva para el tratamiento del cáncer de próstata.

Pacientes y métodos. Se realizó un abordaje extraperitoneal asistidos por el sistema quirúrgico robótico Da Vinci. Los datos prospectivos incluyeron cuestionarios de calidad de vida, demografia básica (peso, altura e índice de masa corporal), niveles de PSA, estadio clínico y suma de Gleason. Las variables intraoperatorias evaluadas fueron duración de la cirugía, pérdida sanguínea estimada (EBL) y complicaciones. Post-operatoriamente se evaluaron los días de estancia en el hospital, días con catéter uretral, histopatología, niveles de PSA, recuperación de la continencia y la potencia.

Resultados. La duración media de la RALP fue de 180 minutos (rango 140-295); todos los procedimientos tuvieron éxito y no se registraron transfusiones sanguíneas ni muertes relacionadas con la cirugía. La EBL media fue 300 cc ( 40 -1100); $97 \%$ de los pacientes fueron dados de alta el primer dia de post-operatorio con $36 \%$ de hematocrito. La duración media del cateterismo uretral fue 7 dias ( 5-21). La tasa de margenes positivos fue del $14 \%$. La continencia a los 6 meses fue del $95 \%$. La supervivencia libre de recidiva bioquímica fue del $95 \%$ al año. Al año $78 \%$ de los pacientes eran potentes( con o sin ayuda farmacológica), $15 \%$ no eran capaces de mantener la erección para penetrar y $7 \%$ necesitaban aún inyecciones intracavernosas.

Conclusión: La prostatectomía radical laparoscópica asistida por robot se caracteriza por un control máximo en cuanto a la resección de tejido, una pérdida de sangre mínima y unos resultados funcionales muy buenos.

Palabras clave: Prostatectomía radical robótica. Da Vinci. Acceso extraperitoneal.

\section{ABSTRACT}

\section{EXTRAPERITONEAL RADICAL PROSTATECTOMY DA VINCI}

Objetive. To report the outcomes of 100 robotically assisted laparoscopic radical prostatectomies (RALPs), a minimally invasive alternative for treating prostate cancer.

Patients and methods. In all patients was used RALP with an extraperitoneal approach assisted by the da Vinci robotic surgical system (Intuitive Surgical, Sunnyvale, CA, USA). Prospective data collection included quality-of-life questionnaires, basic demographics (height, weight and body mass index), prostate specific antigen (PSA) levels, clinical stage and Gleason grade. Variables assessed during RALP were operative duration, estimated blood loss (EBL) and complications, and after RALP were hospital stay, catheter time, pathology, PSA level, return of continence and potency.

Results. The mean (range) duration of RALP was 180 (140-295) min; all procedures were successful, with no intraoperative transfusions or deaths. The mean EBL was $300 \mathrm{~mL}(40-1100) ; 97 \%$ of patients were discharged home on the first day after RALP with a mean haematocrit of 36\%. The mean duration of catheterization was 7 (5-21) days. The positive margin rate was $14 \%$ for all patients. The overall biochemical recurrence free (PSA level $<0.1 \mathrm{ng} / \mathrm{mL}$ ) survival was $95 \%$ at mean follow-up of 9.7 months. There was complete continence at 6 months in $95 \%$ of patients. At 1 year $78 \%$ of patients were potent (with or without the use of oral medications), 15\% were not yet able to sustain erections capable of intercourse, and another $7 \%$ still required injection therapy.

Conclusion. RALP is a safe, feasible and minimally invasive alternative for treating prostate cancer. Our initial experience with the procedure shows promising short-term outcomes.

Keywords: Robotical radical prostatectomy. Da Vinci. Extraperitoneal access.

$\mathrm{H}$ oy en día, el abordaje de una prostatectomía radical puede ser retropúbico abierto, perineal abierto o laparoscópico. La laparoscopia convencional, con una prolongada curva de aprendizaje y una movilidad limitada del instrumental, se ha perfeccionado considerablemente en los tres últi- mos años gracias a la tecnología Da Vinci (técnica asistida por robot) y cada vez se utiliza más. En los Estados Unidos, cerca del $40 \%$ de todas las prostatectomías radicales se realizan con la tecnología Da Vinci, el 2\% con laparoscopia convencional y alrededor del $60 \%$ con abordaje abierto. 


\section{ACCESO EXTRAPERITONEAL}

La elección del abordaje es una decisión importante al planificar una intervención. La mayoría de los equipos con sistemas robotizados elige el abordaje transperitoneal. Esto se debe a que la mayor parte de los grandes centros norteamericanos adoptaron la técnica de Montsouris de París, donde tradicionalmente la intervención quirúrgica era con abordaje transperitoneal. Sin embargo, en algunos centros de laparoscopia convencional y robótica se estableció el acceso extraperitoneal ${ }^{1,2}$. Las ventajas del acceso extraperitoneal son la evitación de las complicaciones intestinales, una posición inclinada de la cabeza mucho menos marcada durante la intervención, ausencia de irritación peritoneal postoperatoria (obstrucción intestinal incompleta paralítica) y aislamiento temporal en caso de un urinoma o de una hemorragia secundaria. El espacio de trabajo extraperitoneal es suficiente y despejado. Se puede llevar a cabo una linfadenectomía convencional sin mayores problemas. Solamente tras la colocación de una malla laparoscópica después de una hernioplastia inguinal o un trasplante renal elegimos un acceso transperitoneal, ya que ya no se puede diseccionar más el espacio extraperitoneal. En los pocos casos en que se requiere una linfadenectomía más amplia, se recomienda también un procedimiento transperitoneal que permita reabsorber la secreción linfática postoperatoria a través del peritoneo.

\section{LA TECNOLOGÍA DA VINCI}

La palabra "robot" procede del checo "robota" que significa esclavo. El telemanipulador Da Vinci funciona según el principio hombre-máquina (maestro-esclavo):

El cirujano se sienta en una consola de mando y trabaja con unos mandos adaptados para dos dedos de cada una de las manos y con varios pedales. Estos movimientos son detectados por un ordenador a través de una serie de sensores y transmitidos mediante una red de cables a los 3 ó 4 brazos instrumentales que están localizados sobre el paciente (Fig. 1). De esta manera pueden adaptarse los diferentes instrumentos (efectores finales) y la óptica a las necesidades de la operación. Gracias a la existencia de un filtro del temblor, que elimina el pulso de la mano humana, se
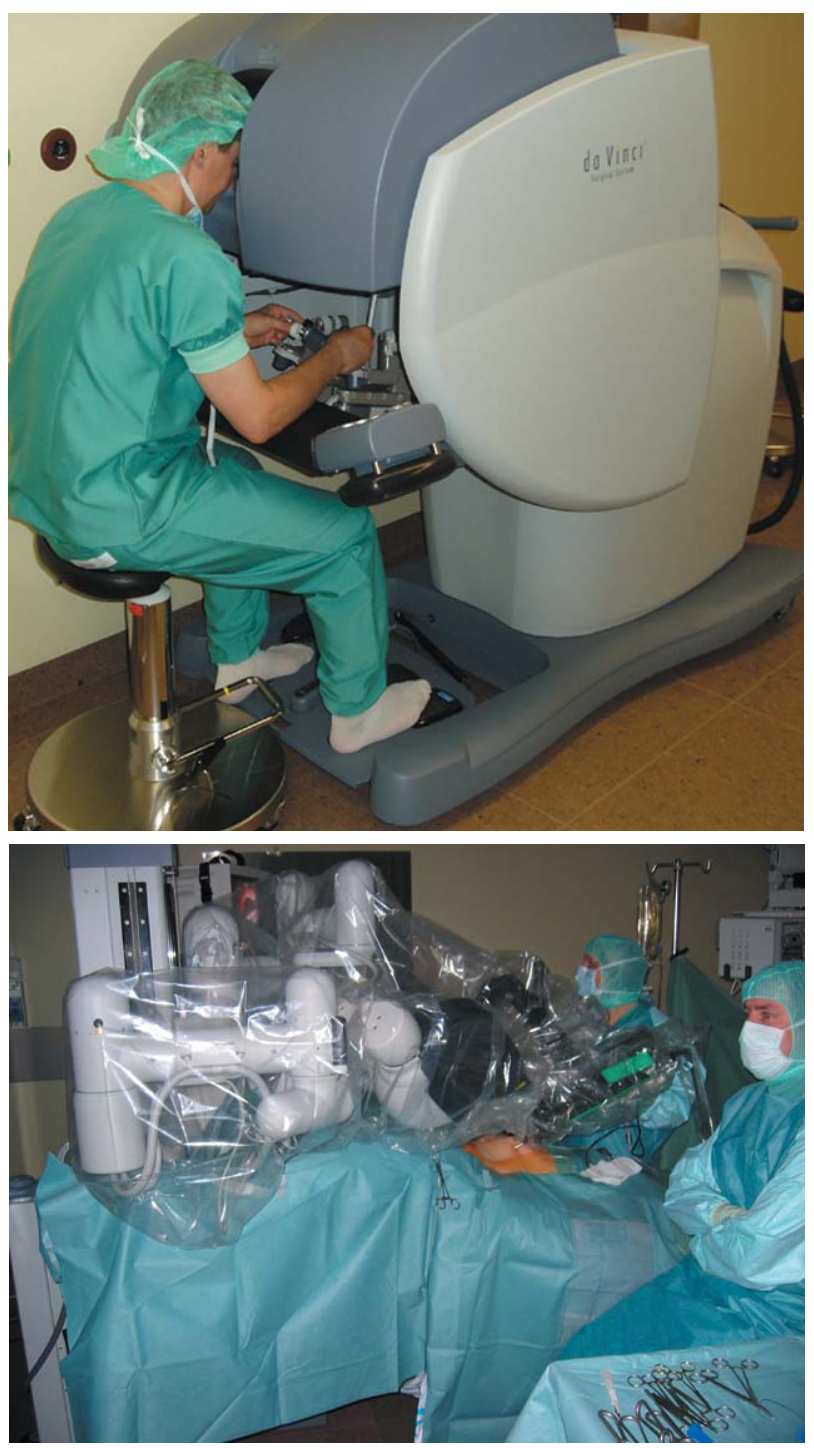

FIGURA 1. Telemanipulador Da Vinci con consola ergonómica (arriba) y brazos instrumentales de precisión en la torre de laparoscopia que poseen los grados de libertad de una mano (abajo).

consigue una gran precisión. La proyección tridimensional y ampliada hasta 10 veces a nivel microscópico del campo de operación en un monitor especial que se encuentra en la consola, así como la posibilidad de escalamiento de los movimientos del instrumental (aumento o disminución), posibilitan una mayor exactitud.

La prostatectomía Da Vinci: Técnica (Fig. 2-12)

En posición de Trendelenburg (cabeza abajo) de 10 a 15 grados, se hace a nivel infraumbilical una incisión transversal de la piel y se abre hasta aproximadamente $1 \mathrm{~cm}$ hasta la fascia rectal 

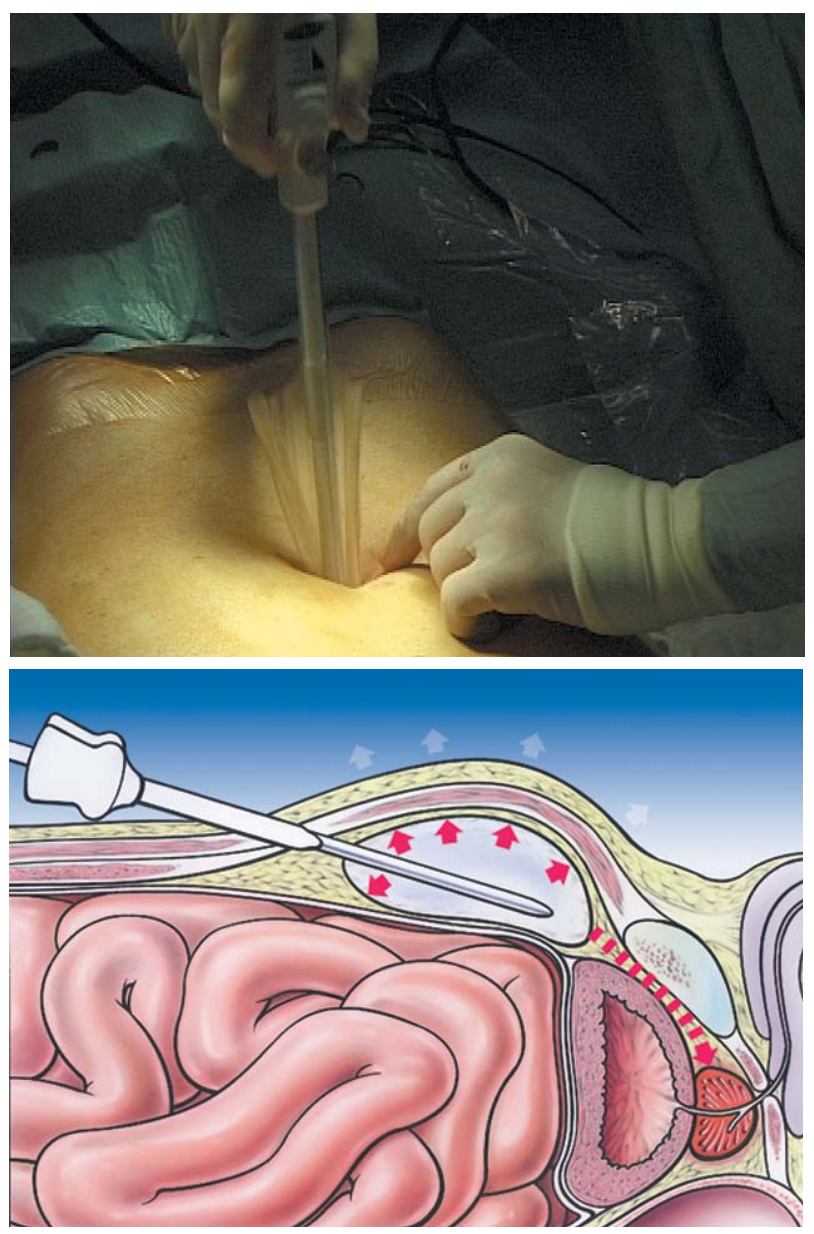

FIGURA 2. El espacio quirúrgico se consigue mediante un balón de dilatación (arriba). De esta manera se consigue el acceso directo a la próstata (abajo).

anterior. Con un separador Langenbeck se expone la aponeurosis abdominal rectal posterior y a lo largo de ella se abre digitalmente el espacio extraperitoneal y a continuación se sigue diseccionando con un trocar de dilatación (Fig. 2). Tras colocar los 5 trocares de trabajo (Fig. 3) en el campo de visión se acoplan los brazos del robot (Fig. 1). En primer lugar se separa la grasa de la superficie anterior de la próstata y de la fascia endopélvica, y se coagula y se corta la vena dorsal por encima del plexo de Santorini. A continuación se abre bilateralmente la fascia endopélvica (Fig. 4), exponiendo el vértice al apartar las fibras musculares transversales del elevador del ano y en dirección distal hasta la región del esfinter. A continuación se liga el plexo de Santorini (Fig. 5). En ese momento se disecan las fibras del cuello vesical del detrusor de la base de la próstata y se expone la porción proximal de la

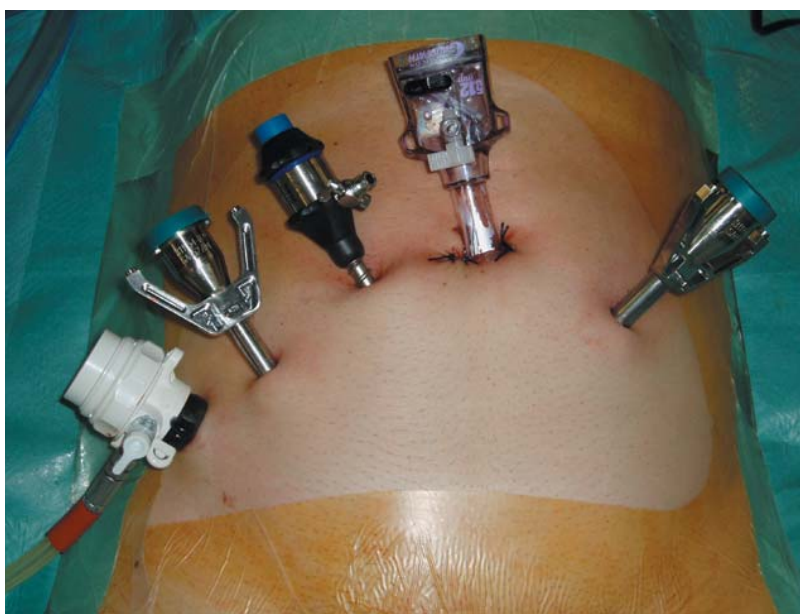

FIGURA 3. En total, se colocan 5 trocares en el espacio extraperitoneal. El acceso de la cámara es infraumbilical y los trocares de trabajo robotizados están colocados en posición paramediana a ambos lados.

uretra (Fig. 6). Tras diseccionar la pared posterior de la uretra se accede al espacio retrovesical y se ligan los conductos deferentes. Conservando los nervios, se disecan las vesículas seminales (Fig. 7). Se retraen los haces neurovasculares en sentido descendente y dorsolateral evitando estrictamente la corriente monopolar y bipolar (Fig. 8-9). Los pedículos prostáticos se sujetan con pinzas y se diseccionan. A continuación se disecciona anteriormente el plexo de Santorini y se expone el muñón uretral. A los lados de éste se encuentran bien diferenciados los haces neurovasculares en todo su recorrido distal. Se diseca la próstata (Fig. 10).

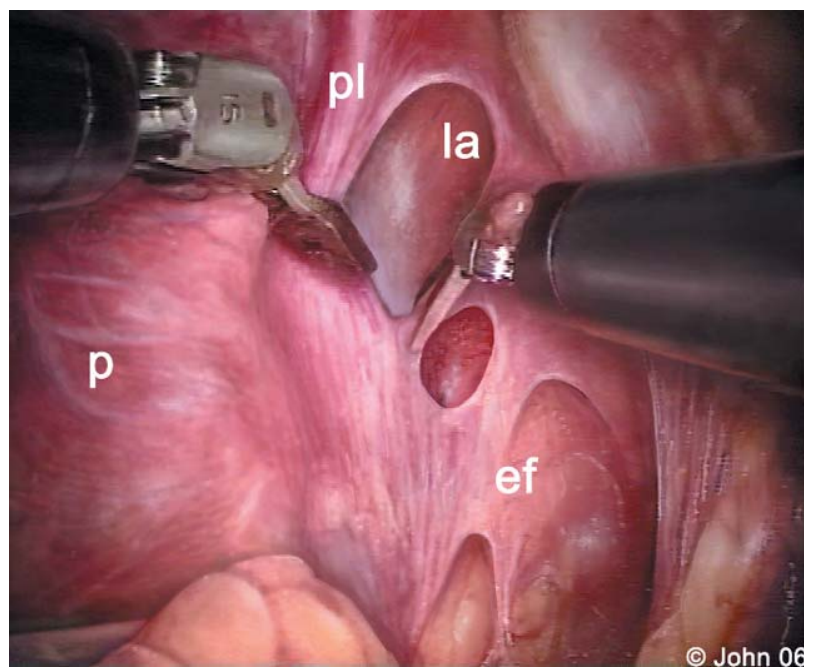

FIGURA 4. Se hace una incisión bilateral de la fascia endopélvica. 


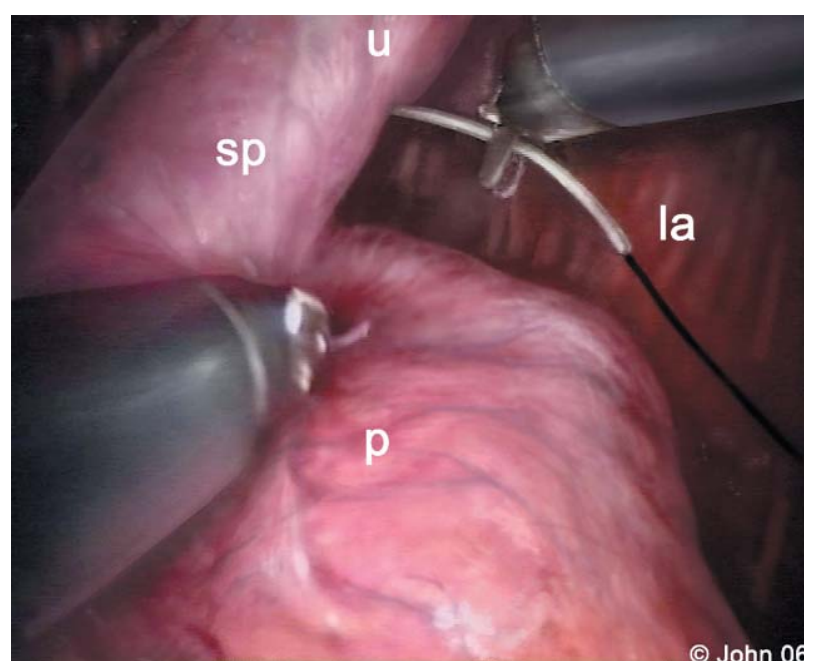

FIGURA 5. Por motivos hemostáticos se liga el plexo de Santorini.

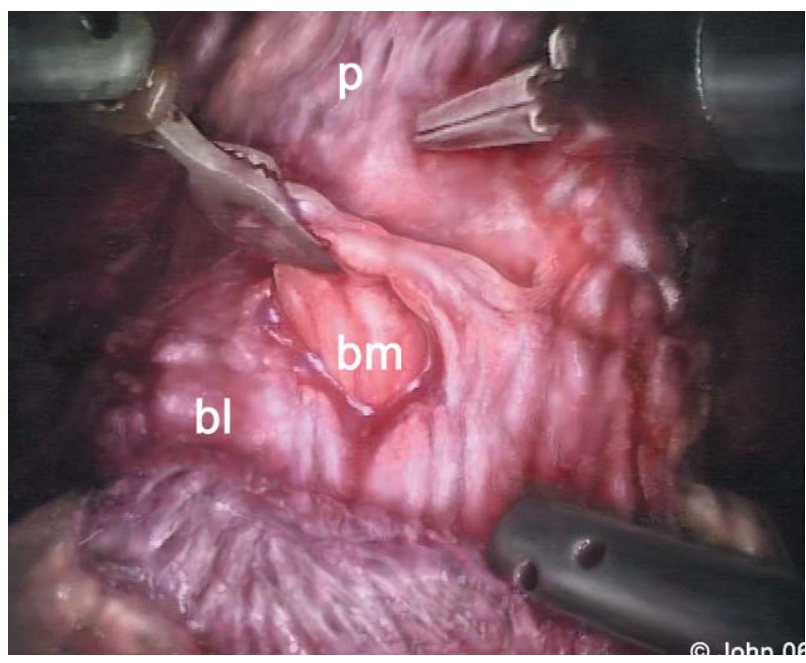

FIGURA 6. El cuello vesical se disecciona cuidadosamente.

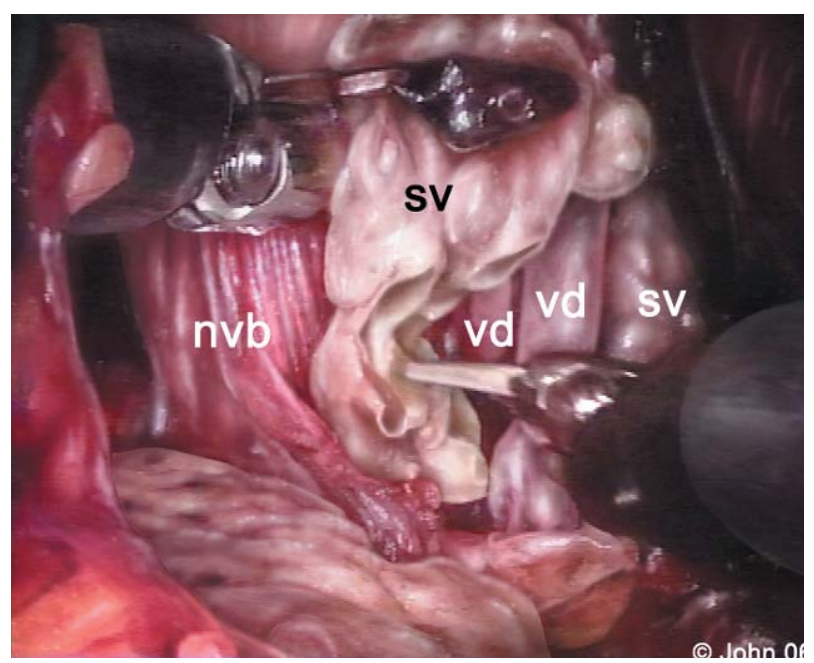

FIGURA 7. Se disecan los anexos.

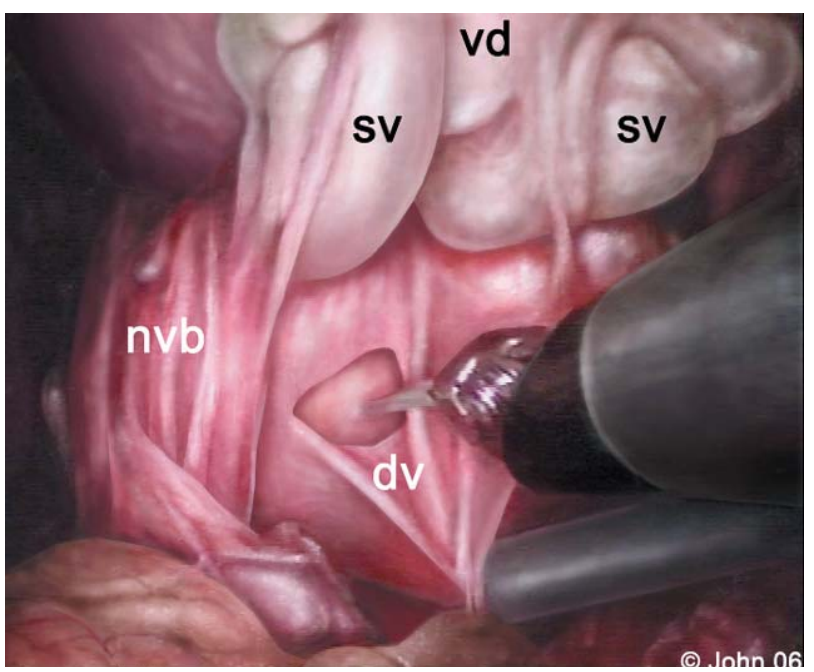

FIGURA 8. Una vez abierta la fascia de Denovillier, se retrae la próstata del recto.

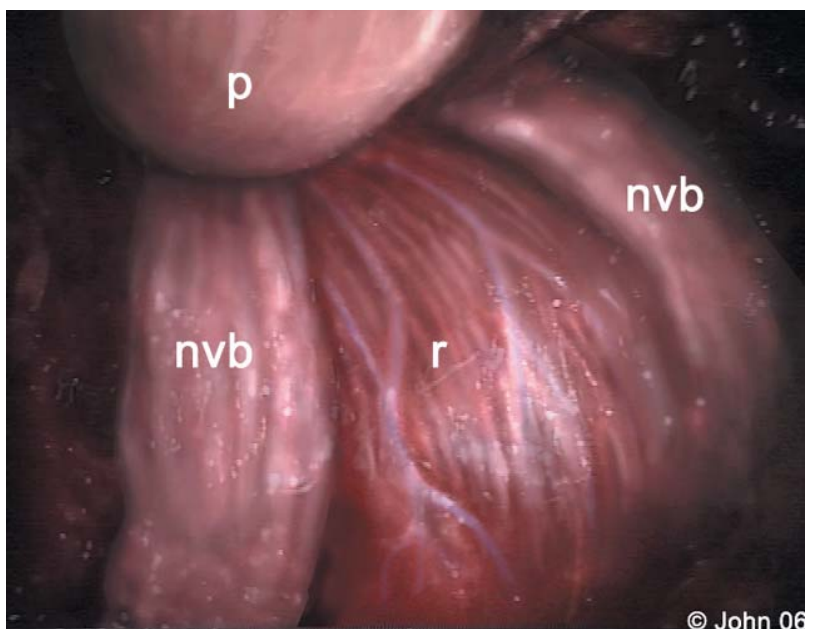

FIGURA 9. Los haces neurovasculares se conservan en la medida de lo posible.

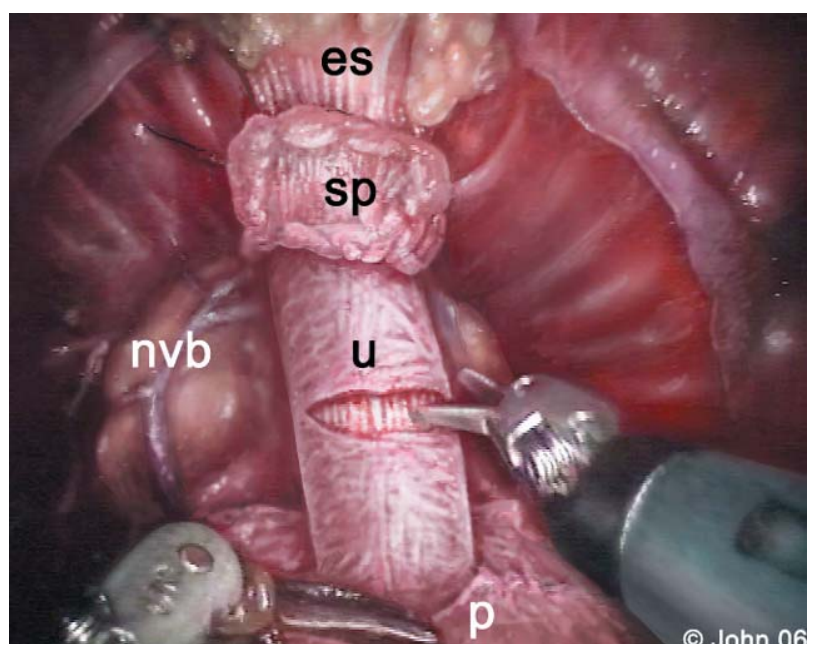

FIGURA 10. En dirección apical se forma un muñón uretral lo más grande posible y se extirpa la pieza. 
La anastomosis se realiza mediante sutura continua o sutura interrumpida. Nosotros preferimos siempre la técnica de sutura interrumpida, más segura, con Vicryl-4-0 y la aguja UR-6 (Fig. 11). Una vez extraída la pieza a través del acceso mediano (Fig. 12) se coloca un drenaje de redón durante 24 horas y una vez transcurrido este tiempo se retira la infusión siempre que haya una buena absorción por vía oral. La analgesia se realiza con paracetamol y como precaución, a menudo se mantiene hasta el segundo día del postoperatorio. La retirada del catéter vesical tiene lugar al séptimo día después de la intervención con el correspondiente protocolo de micción.

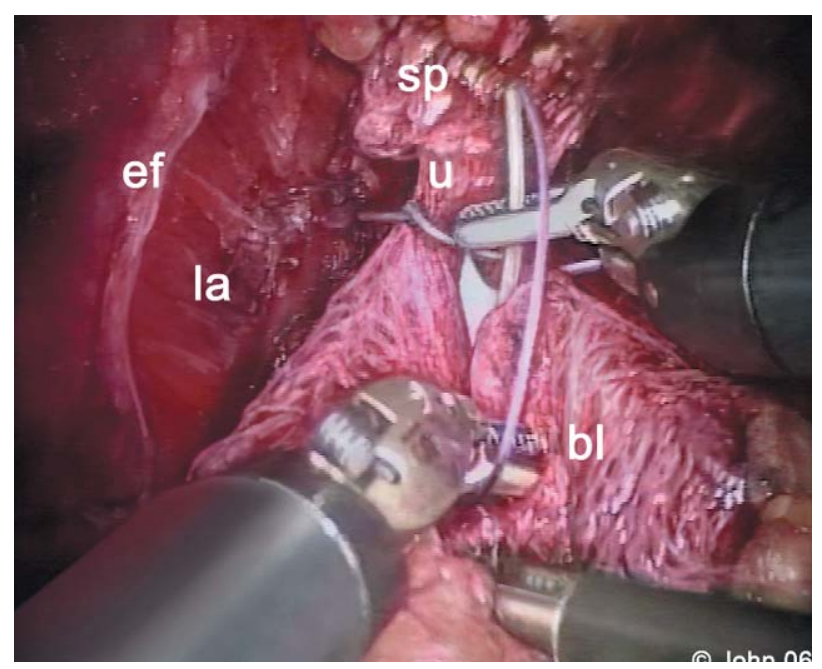

FIGURA 11. Después de realizar una anastomosis vesicouretral.

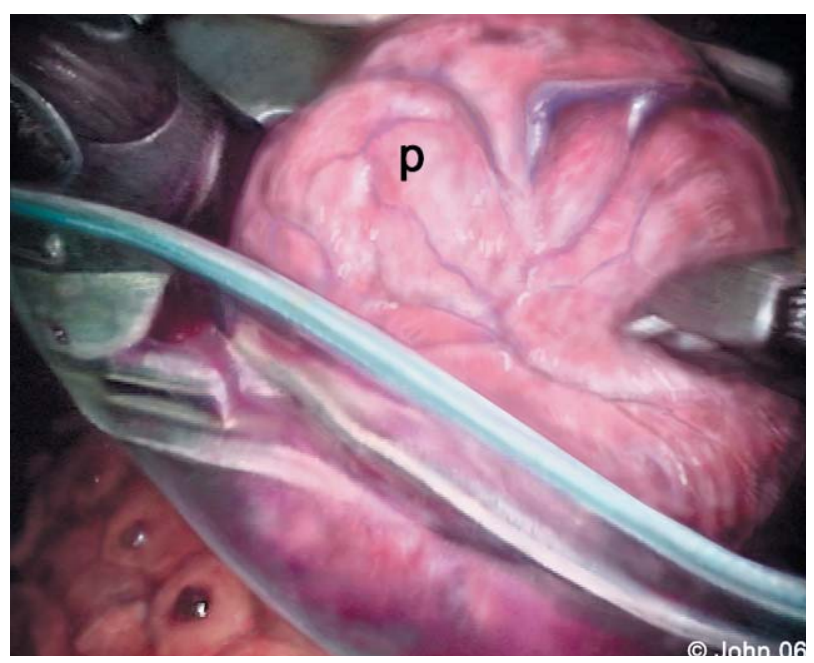

FIGURA 12. Se puede extraer la próstata con una Endobag a través del acceso infraumbilical.

\section{RESULTADOS}

Desde la primera intervención realizada en septiembre de 2002 se ha ido tipificando paulatinamente la técnica Da Vinci y el primer autor ha recogido más de 300 intervenciones Da Vinci (200 en el Universitätsspital Zürich 2002-2005 [1 ${ }^{\text {a }}$ Serie John/Schmid] ${ }^{3}$ y 100 en la Klinik Hirslanden [ $2^{\text {a }}$ Serie, John/Fehr] desde septiembre de 2005). A continuación se incluyen los resultados de la segunda serie (Tablas 1 y 2).

Gracias a la escasa pérdida de sangre (aproximadamente $300 \mathrm{ml}$ ), el tiempo de recuperación del paciente es corto. No es necesario realizar ningún análisis de donante de sangre ajena o de la propia sangre. El porcentaje de continencia (seco sin compresa o una compresa como máximo por seguridad) es del 95\% al cabo de 6 meses. Cuando se conservan ambos haces neurovasculares, la erección con capacidad de penetración se mantiene en el $75 \%$ de los pacientes con ayuda farmacológica. En un seguimiento breve se pudo observar un nuevo aumento del PSA (indicador bioquímico de recidiva tumoral) del 3\% en los tumores limitados al órgano. Los trabajos más recientes de los colegas norteamericanos describen incluso unas tasas de potencia y de continencia de hasta el $98 \%$ al cabo de un año. La incapacidad laboral de los 20 primeros pacientes en edad laboral en la Klinik Hirslanden fue de 21 días como media, mientras que en el caso de la cirugía abierta fue de 80 días. La curva de aprendizaje de un cirujano Da Vinci es considerablemente menor que en el caso de la laparoscopia convencional. Sin embargo, son necesarias como mínimo 100 intervenciones Da Vinci realizadas por un mismo cirujano para poder conseguir los resultados oncológicos y funcionales obtenidos en las mejores series de comparación de cirugía abierta para que los pacientes puedan aprovecharse de esta técnica mínimamente invasiva.

\section{CONCLUSIONES}

La prostatectomía laparoscópica asistida por robot permite realizar una prostatectomía radical curativa con la máxima conservación de tejido, con una pérdida mínima de sangre y una buena conservación de la función eréctil y de la continencia. Por tanto, la recuperación del paciente tras la intervención es corta y permite un regreso rápido al puesto de trabajo. 
Tabla 1

Resultados intra y postoperatorios

\begin{tabular}{|c|c|c|}
\hline Criterio & $\mathbf{N}$ & Resultados \\
\hline \multicolumn{3}{|l|}{ Pacientes } \\
\hline Edad & 100 & 64 (44-76) años \\
\hline PSA & 100 & $6,7(1,2-53) \mathrm{ng} / \mathrm{ml}$ \\
\hline Índice de masa corporal (IMC) & 100 & $27(22-37)$ \\
\hline \multicolumn{3}{|l|}{ Datos quirúrgicos } \\
\hline Duración de la intervención & 100 & 180 (140-295) minutos \\
\hline Pérdida de sangre & 100 & $300(40-1100) \mathrm{ml}$ \\
\hline Conversión & 100 & $0 \%$ \\
\hline \multicolumn{3}{|l|}{ Complicaciones } \\
\hline Lesión rectal & $0 / 100$ & $0 \%$ \\
\hline Lesión ureteral & $0 / 100$ & $0 \%$ \\
\hline Hemorragia secundaria & $2 / 100$ & $2 \%$ \\
\hline Infección de la herida & $0 / 100$ & $0 \%$ \\
\hline Fuga por anastomosis & $2 / 100$ & $2 \%$ \\
\hline Linfocele & $5 / 100$ & $5 \%$ \\
\hline Duración del sondaje & 100 & $7 \mathrm{~d}$ (mediana) \\
\hline Recuperación laboral del 100\% & 20 & 21 días (mediana) \\
\hline \multicolumn{3}{|l|}{ Continencia } \\
\hline Continencia 6 semanas & 81 & $68 \%$ (40\% en la $1^{\underline{a}}$ serie) \\
\hline Continencia 3 meses & 61 & $73 \%$ (53\% en la $1^{\underline{a}}$ serie) \\
\hline Continencia 6 meses & 43 & $95 \%$ (73\% en la $1^{a}$ serie) \\
\hline \multicolumn{3}{|l|}{ Oncología } \\
\hline Volumen tumoral & 100 & $2,65(0,05-58) \mathrm{cm}^{2}\left(\right.$ de los que $\left.3 \%<0,5 \mathrm{~cm}^{2}\right)$ \\
\hline Márgenes positivos & 79 & $14 \%$ \\
\hline Nuevo aumento del PSA & 100 & $\begin{array}{c}3 \% \text { (mediana de seguimiento después de } 6,5 \\
(1-12 \text { meses) }\end{array}$ \\
\hline
\end{tabular}

Tabla 2

Resultados funcionales y oncológicos de los estudios de comparación

\section{Continencia}

\section{Autor}

Ahlering T. et al. ${ }^{4}$

Patel V. et al. ${ }^{5}$

Menon M. et al. ${ }^{6}$

\section{Año}

2004

2005

2006
$\mathbf{N}$

60

200

1452
Continencia (\%)

$76 \%$ (3 meses, sin compresa) 985 (12 meses, sin compresa) 97\% (12meses, sin compresa)

Potencia (conservación intraoperatoria de los nervios, inhibidor de la fosfodiesterasa)

\section{Autor}

Ahlering T. et al. ${ }^{7}$

Patel V. et al. ${ }^{5}$

Menon M. et al. ${ }^{6}$

\section{Año}

2005

2005

2006

\section{$\mathbf{N}$}

23

200

1452
Penetración (\%)

47\% (3 meses)

98\% (12 meses)

75\% (12 meses)

Márgenes positivos (Congreso Norteamericano de Urología 2006)

\section{Autor}

Sarle et al. ${ }^{6}$

Ahlering et al. ${ }^{8}$

Joseph et al. ${ }^{9}$

Zorn et al. ${ }^{10}$

Desai et al. ${ }^{11}$
Año

2006

2006

2006

2006

2006
T2 - márgenes positivos (\%)

$5 \%$

$5 \%$

$15 \%$

$14 \%$

$23 \%$ 


\section{REFERENCIAS}

1. Hoznek A, Antiphon P, Borkowski T, Gettman MT, Katz R Salomon L: Assessment of surgical technique and perioperative morbidity associated with extraperitoneal versus transperitoneal laparoscopic radical prostatectomy. Urology. 2003 Mar;61(3):617-622.

2. Stolzenburg JU, Rabenalt R, Do M, Bekos A, Stief C, Jonas U. Endoscopic ectraperitoneal radical prostatectomy (EERPE)experience after 300cases. J Urol.. 2004:170:217 (abstract 822).

3. John H, Engel N, Brugnolaro C, Muentener M, Strebel R, Schmid DM, Hauri D and Jaeger P: From standard laparoscopic to robotic extraperitoneal prostatectomy: Evolution in 350 cases. Eur Urol. Suppl. 2006;5:117.

4. Ahlering TE, Woo D, Eichel L, Lee DI, Edwards RA and Skarecky DW: Robot-assisted versus open radical prostatectomy: a comparison of on surgeon's experience. Urology. 2004 May;63(5):819-822.

5. Patel VR, Tully AS, Holmes R and Lindsay J: Robotic radical prostatectomy in the community setting - the learning curve and beyond: initial 200 cases. J Urol.. 2005 Jul; 174 (1):269-272.

6. Sarle R, Bhandari A, Shah N, Shrivastava A, Fumo M, Badani K, Kaul S and Menon M: The vattikuti institute prostatectomy: a single surgeon experience of 1452 cases. J Urol.. 2006;174:370-371.
7. Ahlering TE, Eichel L and Skarecky DW: Early potency outcomes with cautery-free neurovascular bundle preservation with robotic laparoscopic radical prostatectomy. $\mathrm{J}$ Endourol.. 2005 Jul-Aug; 19(6):715-718.

8. Ahlering TE, Patel HR, Lee DI and Skarecky DW: Multi-institutional review of pathological margins after robot-assisted laparoscopic prostatectomy (RLP). J Urol. 2006;175: 372 .

9. Joseph JV, Boczko J, Goijanin D, Madeb RR, Vicente I, Erturk E, Rosenbaum RS and Patel HR: Robotic extraperitoneal radical prostatectomy: an alternative approach.. J Urol.. 2006 Mar; 175(3 Pt 1):945-950.

10. Zorn KC, Mikhail AA, Orvieto MA, Chien GW, Zagaja GP and Shalhav AL: Robotic assisted laparoscopic prostatectomy: outcomes of first 300 cases. J Urol.. 2006; 175:370.

11. Desai P, Sundaram CP, Su X, Gardner T and Koch MO: Robotic assited laparoscopic prostatectomy vs.open radical retropubic prostatectomy: characteristics of pathologic positive surgical margin. J Urol. 2006;175:373.

Correspondencia autor: Dr. Hubert John Zentrum für Urologie. Klinik Hirslanden, Zürich

E-mail autor: hubert.john@hirslanden.ch Información artículo: Original 